EPJ Web of Conferences 70, 00071 (2014)

DOI: 10.1051/epjconf/ 20147000071

(C) Owned by the authors, published by EDP Sciences, 2014

\title{
Top quark physics at the LHC
}

\author{
Tae Jeong Kimª for the CMS collaboration \\ ${ }^{1}$ Korea University, Seoul
}

\begin{abstract}
In 2011, an integrated luminosity of more than $5 \mathrm{fb}^{-1}$ at $7 \mathrm{TeV}$ has been delivered by the LHC. The measurement of the cross section in top quark pair production and in single top quark production, top quark mass, top quark properties and new physics searches in top quark decays have been performed at the CMS experiment with various integrated luminosities. An overview of the latest results of these measurements and searches by the time of ICFP 2012 conference will be presented.
\end{abstract}

\section{Introduction}

The CMS experiment [1] at the Large Hadron Collider (LHC) has accumulated data corresponding to an integrated luminosity of $5 \mathrm{fb}^{-1}$ in 2011. At the LHC, the $t \bar{t}$ production cross section at $\sqrt{s}=7$ $\mathrm{TeV}$ is predicted to be $164.6 \mathrm{pb}$ by approximate next-to-next-leading-order (NNLO) calculations and $157.5 \mathrm{pb}$ by next-leading-order (NLO) calculations. The cross section measurement of $t \bar{t}$ is important for testing perturbative QCD, which is successful so far and searching for new physics. Any deviation from predictions would indicate possible new physics. It is crucial to measure the cross section in all decay modes since new physics can appear in any different decay modes. The top quark decays almost exclusively to a $W$ boson and a $b$ quark. Therefore, the decay mode entirely depends on the $W$ boson branching ratio. In the dilepton decay mode (ee, $e \mu, \mu \mu)$, the tau leptonic decay is included. Considering $\operatorname{Br}\left(\tau \rightarrow l v_{l} v_{\tau}\right)=0.35$, the branching ratio is $6.8 \%, 3.8 \%, 9.6 \%, 30 \%$ and $44 \%$ for dilepton, tau+lepton, tau+jets, lepton+jets and all hadronic decay modes, respectively. In addition to inclusive cross section, measuring the cross section differentially is also important to test the perturbative QCD since any deviation can arise in different kinematic phase space. As a deviation in top quark forward-backward asymmetry has been observed by Tevatron, the charge asymmetry measurement at the LHC are also performed using the fact that the width in rapidity distribution of top quark is slightly broader than anti-top quark at the LHC. An overview of the latest results of these measurements and searches by the time of ICFP 2012 conference will be presented.

\section{Samples \& Objects}

The signal sample of $t \bar{t}$ is modeled by MADGRAPH with PYTHIA matching up to three additional partons. An NLO cross section of $157.5 \mathrm{pb}$ is used for normalization. The $\tau$ decay is handled by TAUOLA and the top quark mass is assumed to be $172.5 \mathrm{GeV}$. In top quark analyses, almost all

a e-mail: Tae.Jeong.Kim@cern.ch

This is an Open Access article distributed under the terms of the Creative Commons Attribution License 2.0, which permits unrestricted use, distribution, and reproduction in any medium, provided the original work is properly cited. 
physics objects are used. The physics objects are reconstructed through a particle-flow reconstruction algorithm which combines information from all sub-detectors and reconstruct all particles. The $|\eta|$ of electrons and muons are required to be within 2.5 and 2.4, respectively. Tau is identified with the Hadrons plus strips (HPS) algorithm combining charged hadrons and calorimeter information in strips to take into account $\pi^{0}$ [2]. Particle-flow jets are reconstructed using anti-kt algorithm with $\mathrm{R}=0.5$. $E_{T}^{m i s s}$ is the opposite direction of vector sum of reconstructed particles.

\section{Inclusive $t \bar{t}$ cross section measurements}

The dilepton decay mode (ee, $\mathrm{e} \mu, \mu \mu$ ) provides clean signals by requiring two isolated leptons with two jets and $E_{T}^{\text {miss }}$ even though the branching ratio is small. CMS performed the analysis with an integrated luminosity of $1.1 \mathrm{fb}^{-1}$ [3]. The invariant mass of dilepton must be above $12 \mathrm{GeV}$ and $\left|M_{l l}-M_{Z}\right|>15 \mathrm{GeV} . E_{T}^{\text {miss }}>30 \mathrm{GeV}$ is required for $e e$ and $\mu \mu$ decay modes. At least one $b$-tagged jet is required. Lepton efficiencies are obtained with $Z$ boson candidates in a data-driven way. DrellYan and multi-jet backgrounds are also obtained in a data-driven way. Distributions of the number of jets for all channels combined after the dilepton pair requirement and the number of $b$-tagged jets for all channels combined after the requirement of missing transverse energy are shown in Fig. 1. The cross section for each decay mode is obtained using a counting method. The combined result of the three decay modes is found to be

$$
\sigma_{t \bar{t}}=169.9 \pm 3.9 \text { (stat.) } \pm 16.3 \text { (syst.) } \pm 7.6 \text { (lumi.) } \mathrm{pb}
$$

using the Best Linear Unbiased Estimator (BLUE) method. The measured cross sections are consistent with NNLO predictions.

The $\mu+\tau$ decay mode is interesting since the charged higgs can decay with the same topology when the higgs mass is larger than top mass. Any deviation on cross section would indicate the new physics. Therefore, reducing the systematic uncertainty is crucial in this decay mode. This analysis follows lepton+jet analysis event selection since hadronic tau decay is considered. The analysis is performed with an integrated luminosity of $1.1 \mathrm{fb}^{-1}$ data [7]. The tau fake rate from jets is estimated from multi-jet (gluon jet) and $W+$ jets (quark jet) data samples. The measured cross section is

$$
\left.\sigma_{t \bar{t}}=148.7 \pm 23.6 \text { (stat.) } \pm 26.0 \text { (syst. }\right) \pm 8.9 \text { (lumi.) pb. }
$$

The main systematic uncertainties are from tau fake background estimation, identification and $b$ tagging efficiency.

The cross section in $\tau+$ jets decay mode is also measured with $3.9 \mathrm{fb}^{-1}$ [6]. Events are required to have at least 4 jets. The main background, multi-jet events, is obtained from data. Using a Neural Network (NN) method, the cross section is measured to be

$$
\sigma_{t \bar{t}}=156 \pm 12 \text { (stat.) } \pm 33 \text { (syst.) } \pm 3 \text { (lumi.) pb. }
$$

In the lepton+jet decay mode $(\mathrm{e}, \mu)$, the signature of a final state is one exclusive lepton, 4 jets and $E_{T}^{\text {miss }}$. The analysis is performed with an integrated luminosity of $1.1 \mathrm{fb}^{-1}(\mu)$ and $0.8 \mathrm{fb}^{-1}$ (e) [8]. Exclusively one isolated muon or electron must have $p_{T}>35$ or $45 \mathrm{GeV}$, respectively. $E_{T}^{\text {miss }}$ is required to be larger than 20 and $30 \mathrm{GeV}$ for e and $\mu$, respectively. $b$-tagging with a secondary vertex algorithm is required. The multi-jet shapes are obtained from data directly using non-isolated leptons. Binned profile likelihood fitting is applied to secondary vertex mass distribution in $1 b$-tagged and $2 b$-tagged jet bins. The measured cross section is found to be

$$
\sigma_{t \bar{t}}=164.4 \pm 2.8 \text { (stat.) } \pm 11.9 \text { (syst.) } \pm 7.4 \text { (lumi.) pb. }
$$



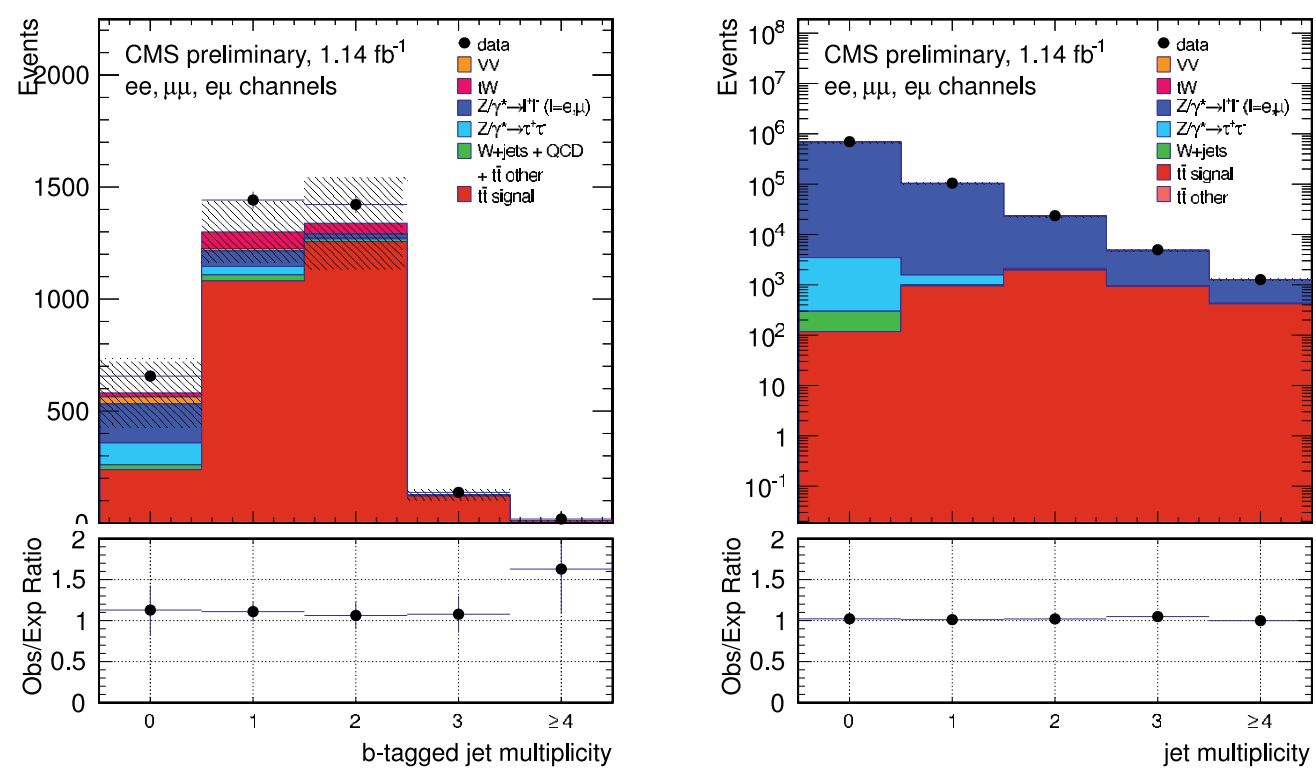

Figure 1. Distribution of the number of jets for all channels combined (left) after the dilepton pair requirement and the number of $b$-tagged jets for all channels combined (right) after the requirement of missing transverse energy. The hatched area represents the uncertainty coming from data-driven background estimates, the scale factors and the luminosity.

The main systematic uncertainties are from $W+$ jets $\mathrm{Q}^{2}$ scale, $b$-tagging efficiency and JES. Comparing the result with $36 \mathrm{pb}^{-1}$ in 2010 [9]-[10], the statistical uncertainty is considerably reduced and the systematic uncertainty is now dominant.

In the hadronic decay mode, the branching ratio of hadronic decay mode is as large as $45 \%$. However, it suffers from large multi-jet background. In the corresponding analysis, 6 jets and at least two $b$-tagged jets are required. CMS performed the analysis with an integrated luminosity of $1.1 \mathrm{fb}^{-1}$ [11]. The multi-jet shape is obtained from data extrapolating from non $b$-tagged jet sample (more than 6 jets) to $b$-tagged jets. In order to take into account the kinematic phase space difference, a scale factor is applied to non $b$-tagged jet sample as a function of $p_{T}$ and $\eta$. Unbinned maximum likelihood fitting is applied to top mass distribution to extract the number of signal. The result of the fit to the reconstructed top mass is shown in Fig. 2. The measured cross section is found to be

$$
\sigma_{t \bar{t}}=136 \pm 20 \text { (stat.) } \pm 40 \text { (syst.) } \pm 8 \text { (lumi.) pb. }
$$

The uncertainties are mainly from $b$-tagging, JES, multi-jet background estimation.

CMS combined the dilepton (ee, e $\mu, \mu \mu, \mu \tau)$, lepton+jets $(\mathrm{e}, \mu)$ and all hadronic decay analysis performed with around $1 \mathrm{fb}^{-1}$ [12]. The binned maximum likelihood fitter from lepton+jet analysis is used for combination adding other decay modes as a single bin. Combined cross section result at CMS comparing to the approximate NNLO calculations are shown in Fig. 3. The combined result is found to be

$$
\sigma_{t \bar{t}}=165.8 \pm 2.2 \text { (stat.) } \pm 10.6 \text { (syst.) } \pm 7.8 \text { (lumi.) pb. }
$$




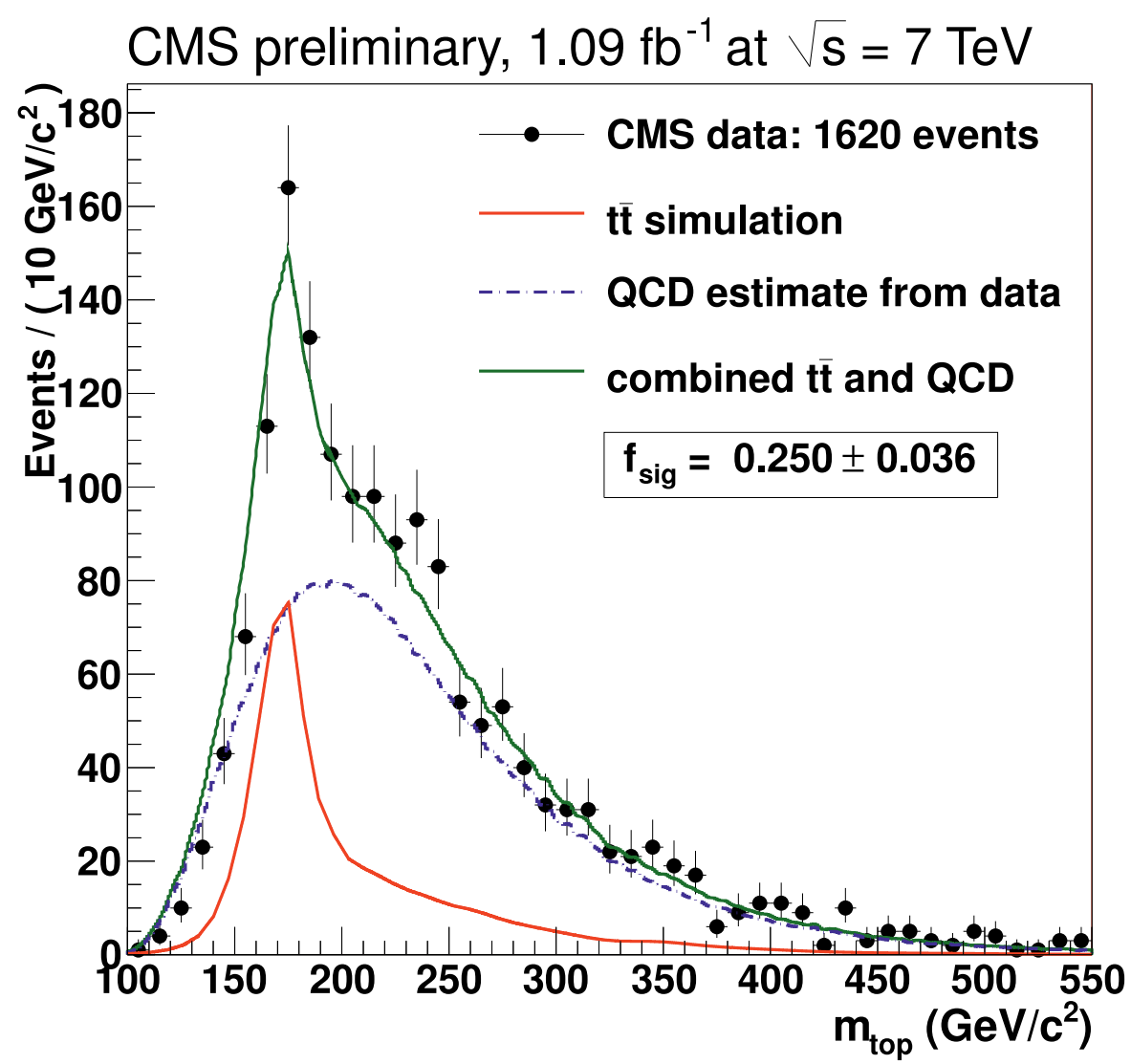

Figure 2. Result of the fit to the reconstructed top mass distribution in hadronic decay mode at CMS.

The total uncertainty in combined analysis at CMS only is obtained to be $8 \%$, which is the most precise measurement at the LHC.

\section{Differential cross section}

Measuring the cross section differentially is important to test the perturbative QCD. Any deviation can arise in different kinematic phase space even though there is a good agreement in inclusive measurements. The differential cross sections are measured extensively as a function of the kinematic properties of the leptons from top quark decays, of top quarks themselves and of the $t \bar{t}$ system in dilepton and lepton+jet decay mode with around $1 \mathrm{fb}^{-1}$ data [13]. The differential cross sections are measured in a visible phase space corresponding to the kinematic and geometric accepted region where all reconstructed final state objects are produced. Unfolding is used to take into account the detector effects correcting to parton level. The measured differential cross section distributions are normalized to unity divided by the total cross section in the visible phase space region to reduce the systematic uncertainties affecting the normalization. The distributions of the transverse momentum of 


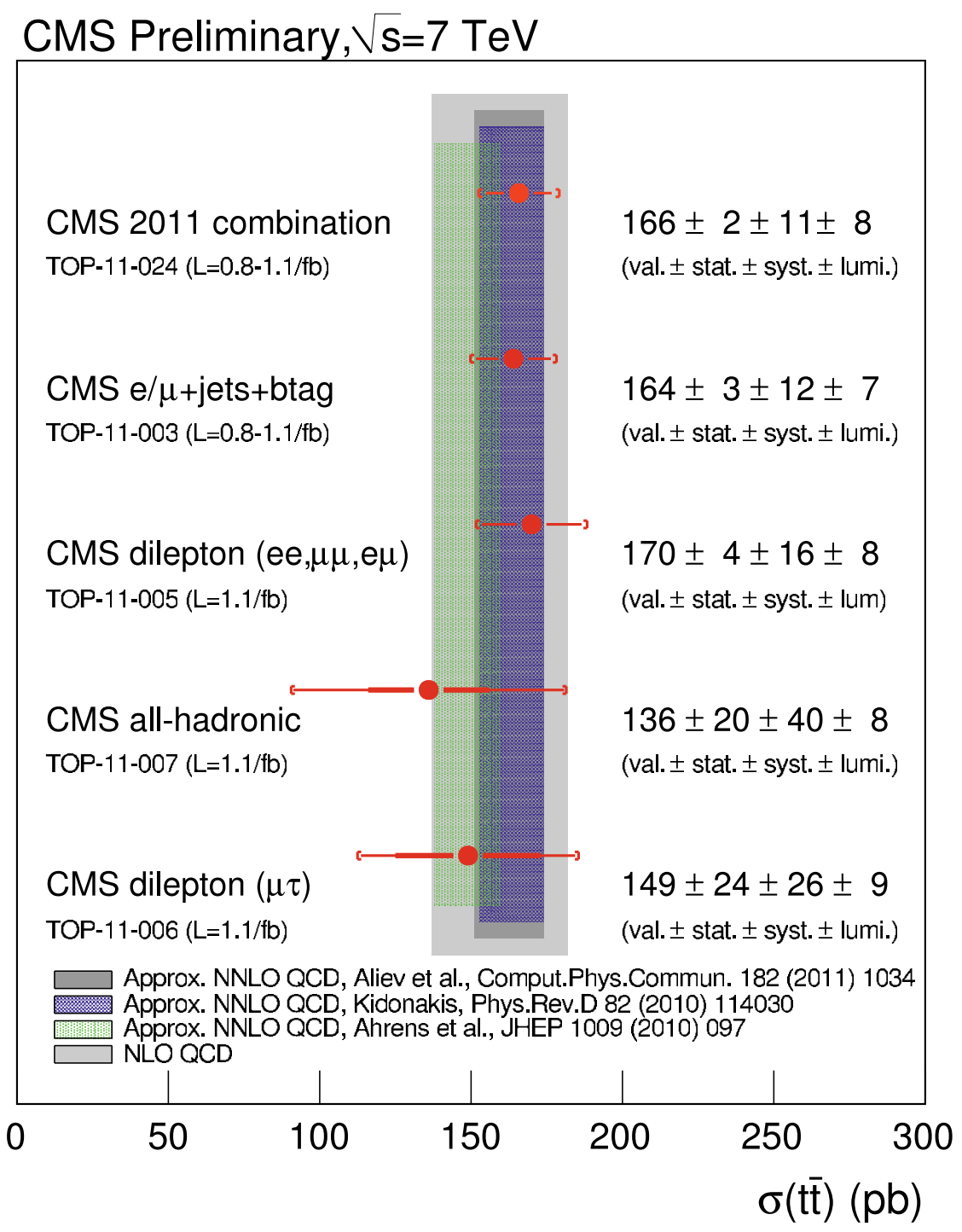

Figure 3. Combined cross section result at CMS. The data are compared to the approximate NNLO calculations performed using the pole mass of top quark $\mathrm{M}_{t}^{\text {pole }}=172.5 \mathrm{GeV}$.

the leptons, top quark and invariant mass of the $t \bar{t}$ system in lepton+jet and dilepton decay modes are shown in Fig 4. The results are compared with various theory predictions and consistent with the SM predictions. 

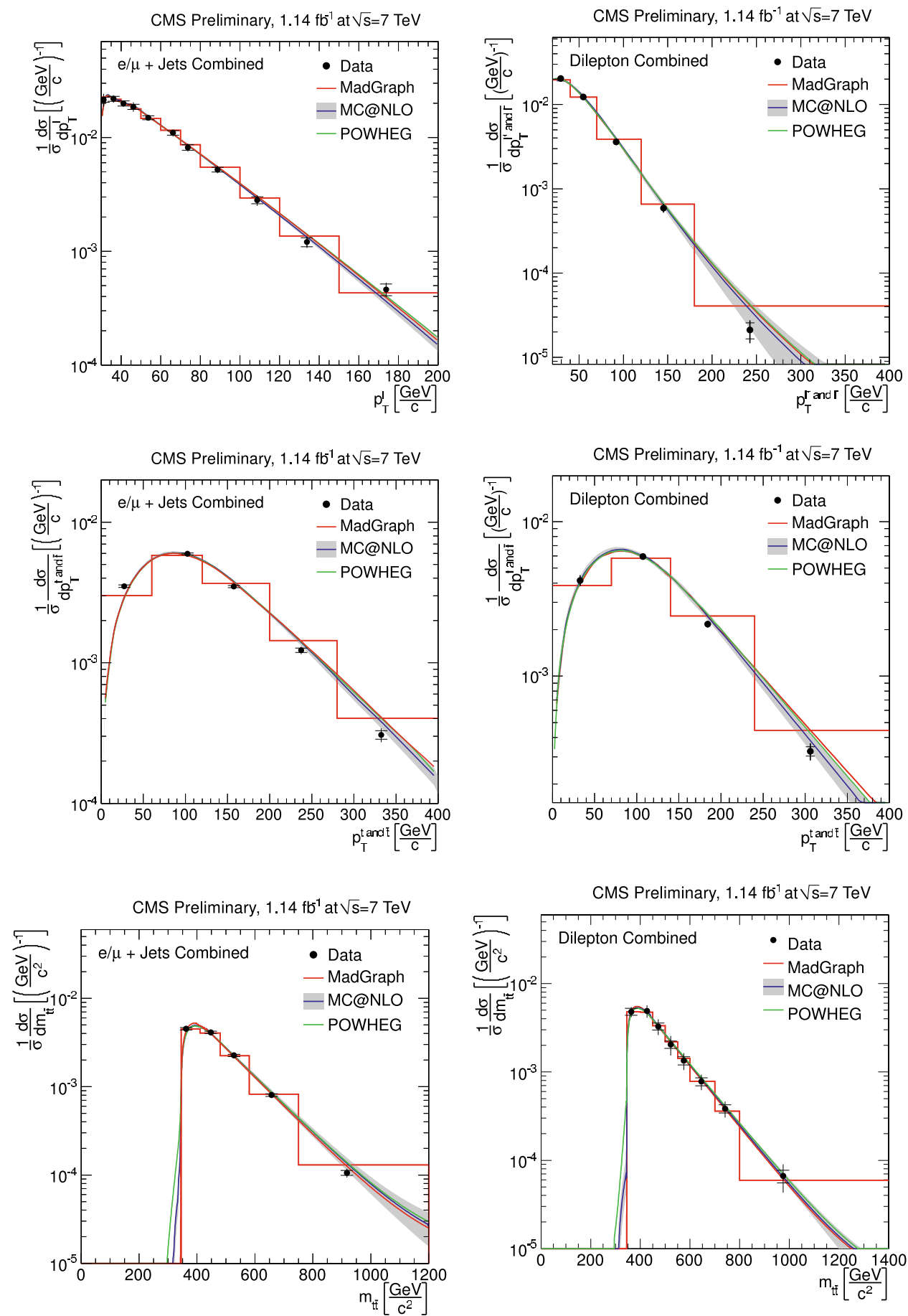

Figure 4. Normalized differential $t \bar{t}$ production cross sections as a function of the transverse momentum of the leptons (upper), top quark (middle) and invariant mass of the $t \bar{t}$ system (lower) in lepton+jet decay mode (left) and in dilepton decay mode (right). The data point is also bin-center corrected with respect to MADGRAPH simulation curve. 


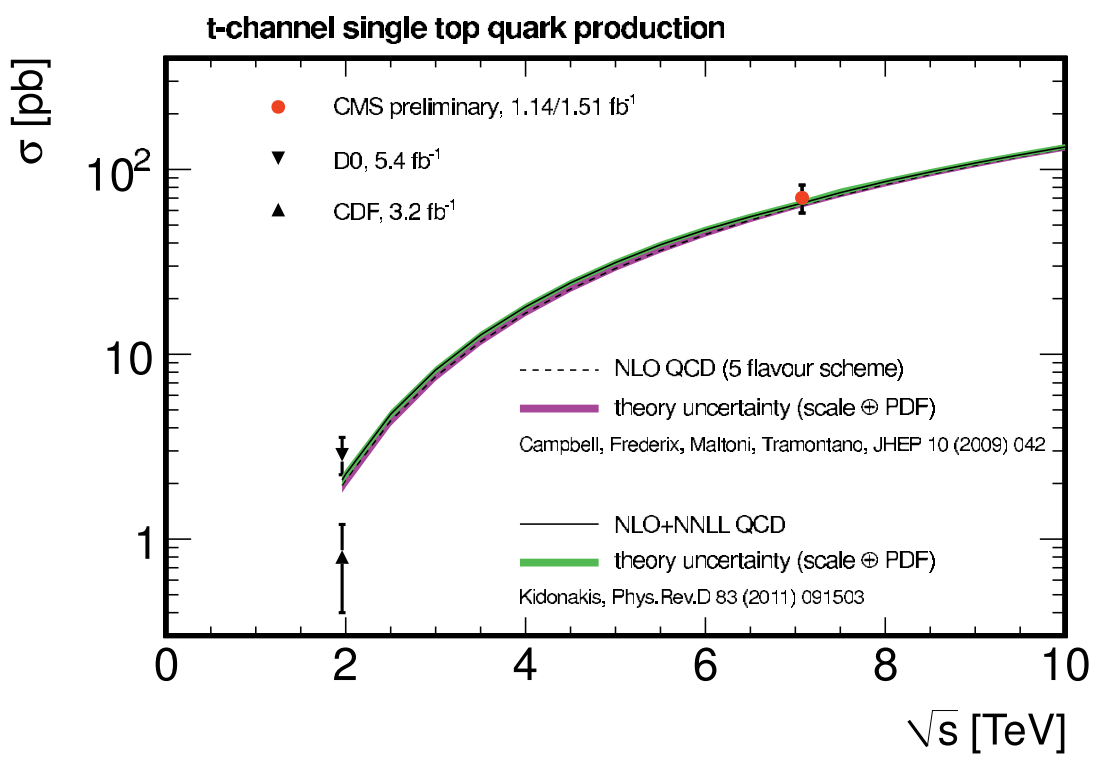

Figure 5. Single top cross section in the $t$-channel versus center of mass energy comparing with Tevatron results.

\section{Single top cross section}

At the LHC, the single top cross section is predicted to be $64 \mathrm{pb}$ for t-channel, $15.6 \mathrm{pb}$ for $t W$-channel and $4.6 \mathrm{pb}$ for s-channel. Analyses in t-channel with around $1 \mathrm{fb}^{-1}$ and in $t W$-channel with around 2 $\mathrm{fb}^{-1}$ are performed.

In t-channel, events are required to have one isolated electron or muon, at least two jets, MET $>30$ $\mathrm{GeV}$ and transverse $W$ boson mass $>40 \mathrm{GeV}$ [14]. The signal fraction is extracted by a fit to angular distribution of pseudo-rapidity of light jet since the jets in the signal events tend to go to forward region. The single top cross section in t-channel is measured to be $70.2 \pm 5.2$ (stat.) \pm 10.4 (syst.) \pm 3.4(lumi.)pb. Figure 5 shows the comparison of the current measurement with the SM prediction as a center of mass energy. A direct measurement of $\left|V_{t b}\right|$ can also be performed in this channel. The $\left|V_{t b}\right|$ Cabibbo-Kobayashi-Maskawa (CKM) element is measured to be $1.04 \pm 0.09$ (exp.) \pm 0.02 (th.)

In $t W$-channel, the event selection is similar to the inclusive cross section analysis in dilepton decay mode since the final state is similar [15]. Additionally the second $b$-tagged jet is vetoed and the transverse momentum of $t \bar{t}$ system is required to be larger than $60 \mathrm{GeV}$. The signal is obtained by using a maximum likelihood fit to the three categorized bins: 1 . one jet and one tagged jet, 2 . two jets and one tagged jet, 3. two jets and two tagged jets. Event yields in data and simulation in the signal region ( 1 jet, $1 \mathrm{tag})$ and the two $t \bar{t}$-enriched control regions for all channels combined are shown in Fig 6. The cross section is measured to be $22_{-7}^{+9}($ stat $\oplus$ syst $)$ pb. The observed significance is $2.7 \sigma$.

\section{Top quark mass}

The top quark mass is measured in muon+jets decay mode with $4.7 \mathrm{fb}^{-1}$ data and in dilepton decay mode with $2.3 \mathrm{fb}^{-1}$ data. In muon+jets decay mode, the likelihood method is used considering all jet 


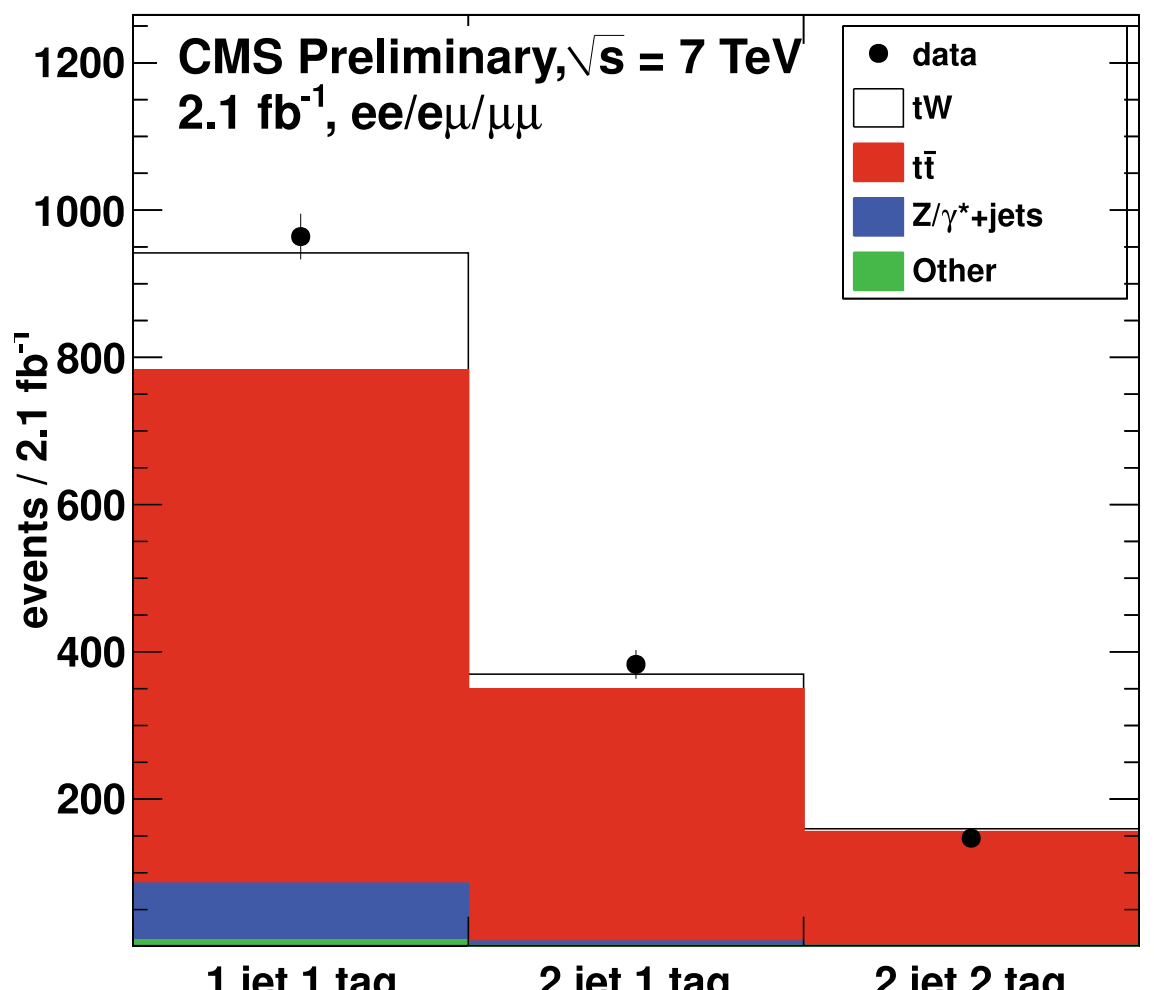

Figure 6. Event yields in data and simulation in the signal region ( 1 jet, 1 tag) and the two $t \bar{t}$-enriched control regions for all channels combined. The Drell-Yan contribution is estimated from data and $t \bar{t}$ contribution is scaled to the outcome of the statistical fit.

permutations and $b$-tagging information to reconstruct four-momentum of top quark [16]. Top quark mass and jet energy scale are obtained simultaneously to reduce the systematic uncertainty. The heavy flavor jet energy uncertainty is found to be dominant uncertainty source in this measurement. In dilepton decay mode, the top quark is reconstructed based on the KINb method [17]. In order to reconstruct the event, the jet $p_{T}$, the $E_{T}^{\text {miss }}$ direction and the longitudinal momentum of $t \bar{t}$ system are varied according to their resolutions to scan the kinematic phase space compatible with the $t \bar{t}$ system. By using the $b$-tagging information, the lepton-jet pair is correctly assigned in $75 \%$ of the cases when the two $b$-jets from the top quark decays have been reconstructed and passed the selection criteria. The systematic uncertainty on the jet energy scale correction is dominant in this decay mode. Combining the results with BLUE method from the results in both decay modes with 2011 data as well as with 2010 data, the combined result is obtained [18]:

$$
M_{t}=172.6 \pm 0.4 \text { (stat.) } \pm 1.2 \text { (syst.) } \mathrm{GeV}
$$




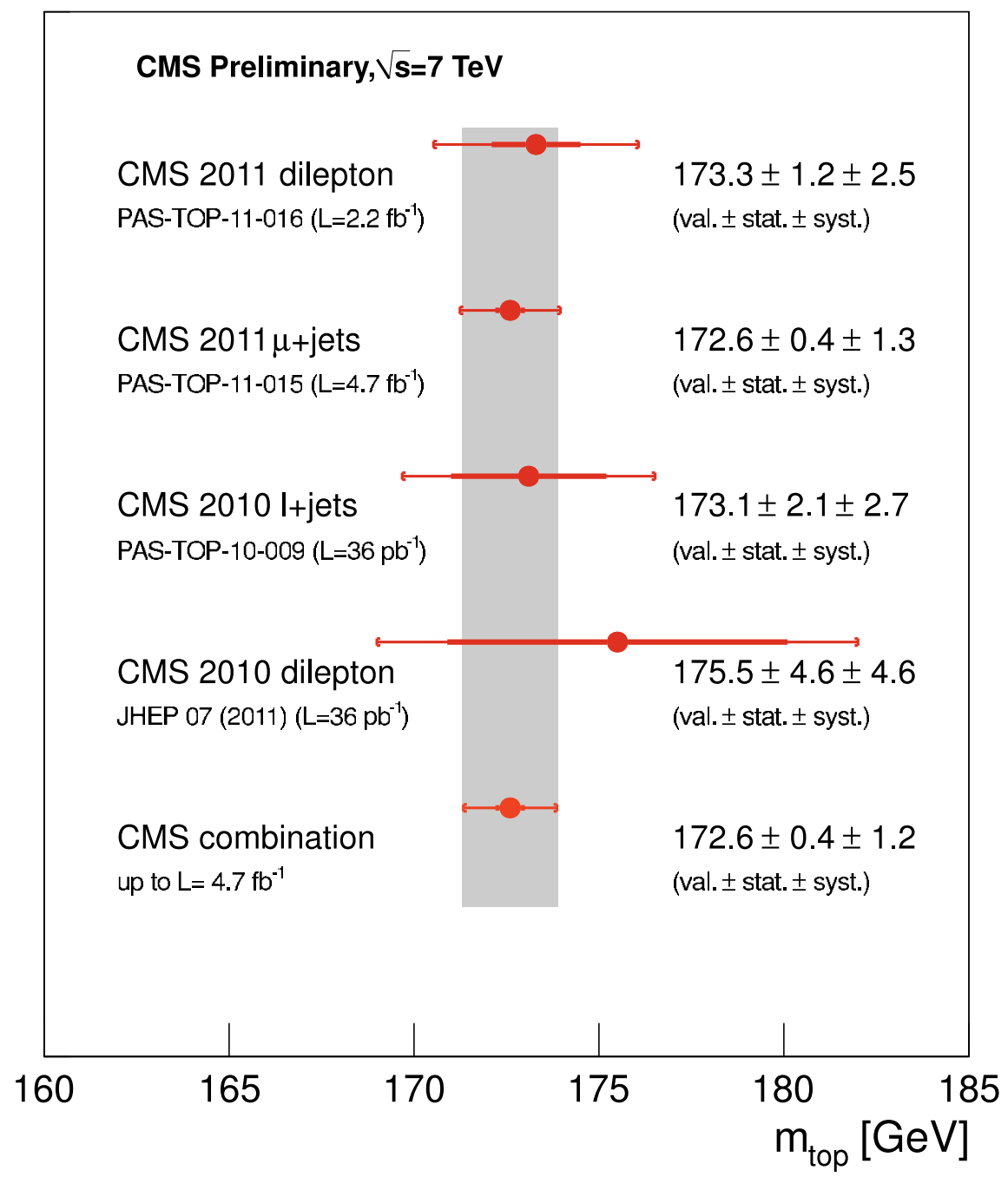

Figure 7. Summary of $\mathrm{CMS} \mathrm{M}_{t}$ measurements and their combination.

The result in each decay mode and the combined result are shown in Fig. 7. The combined result is competitive with Tevatron result.

A measurement of top mass from cross section is also performed with the cross section result from dilepton decay mode [19]. The pole mass and MS mass are extracted from joint likelihood fitting. The measured cross section together with its dependence on the $M_{t}^{M C}$ assumption and approximate NNLO predictions are presented in Fig. 8. The measured cross section corrected for the extracted $M_{t}^{\text {pole }}$ is 


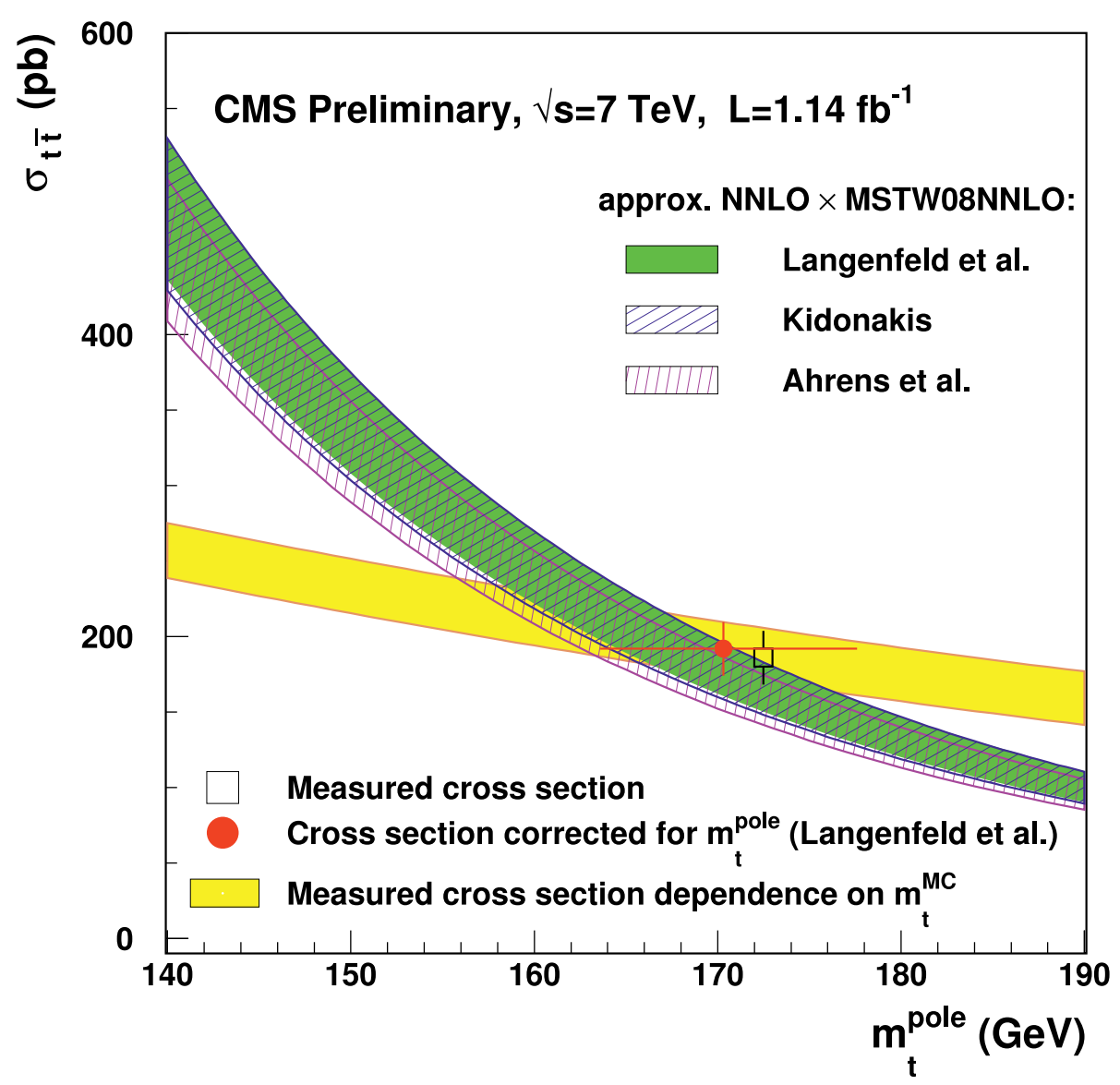

Figure 8. The closed circle indicates the measured cross section corrected for the $M_{t}^{\text {pole }}$ dependence at the determined value of $M_{t}^{\text {pole }}$. The uncertainty on the theoretical predictions includes the variation of the scales, parton luminosity and the variation of the $\alpha_{S}\left(M_{Z}\right)$ in the PDF.

also shown. The dependence on the PDFs is around $1-2 \mathrm{GeV}$. The extracted top quark mass is not competitive with the direct measurement.

\section{$7 t$ and $\bar{t}$ mass difference}

CPT appears to be conserved in nature. As a consequence, the mass of any particles must be equal to that of its anti-particles. A measurement of the mass difference between the top and the anti-top quark is performed using events with a muon or an electron with $5.0 \mathrm{fb}^{-1}$ [20]. The events in this analysis are required to have at least four jets in the final state. The same kinematic fitting method as for top mass reconstruction in lepton + jet decay mode is used. The measured $\Delta M_{t}$ is $-0.44 \pm 0.46$ (stat.) \pm 0.27 (syst.) GeV. This result is the world's best measurement so far. 


\section{FCNC search}

New physics beyond the SM could enhance the $t \rightarrow Z q$ decay mode. A search is performed for $t \rightarrow Z q$ in $t \bar{t} \rightarrow Z q+w b \rightarrow l l j+l v b$ events with data corresponding to $4.6 \mathrm{fb}^{-1}$ [21]. Therefore, the event is required to have three leptons exclusively, at least two jets and MET $>30 \mathrm{GeV}$. The requirement of $\mathrm{HT}_{s}>250 \mathrm{GeV}$ or one $b$-tagged jet is further applied separately. The final events are selected within a mass window (10-250 GeV/c ${ }^{2}$ ) of $M_{Z j}$ and $M_{W j}$ with $\mathrm{HT}_{s}>250 \mathrm{GeV}$ requirement. The mass window of $\left|M_{t}-M_{Z j}\right|<25 \mathrm{GeV}$ and $\left|M_{t}-M_{W j}\right|<35 \mathrm{GeV}$ are applied with one $b$-tagged jet requirement. The branching ratio of $t \rightarrow Z q$ larger than $0.34 \%$ is excluded at $95 \%$ confidence level.

\section{Measurement of $\mathbf{B}(t \rightarrow W b) / \mathbf{B}(t \rightarrow W q)$}

In the SM, the top quark decays to a $W$ boson and a $b$ buark roughly $99.8 \%$ of the time. The magnitude of the CKM matrix element $\left|V_{t b}\right|$ is expected to be close to unity as a consequence of unitarity and of the measured values for the other CKM elements. A deviation from this prediction could arise from a fourth quark family. Measurement of $\mathrm{B}(t \rightarrow W b) / \mathrm{B}(t \rightarrow W q)$ is performed based on $2.2 \mathrm{fb}^{-1}$ data in dilepton decay mode [22]. Events are required to have two isolated leptons and MET $>30 \mathrm{GeV}$ for the ee and $\mu \mu$ decay mode. The fraction $R, \mathrm{~B}(t \rightarrow W b) / \mathrm{B}(t \rightarrow W q)$, is obtained by factorizing the $b$-tagged jet multiplicity as a function of $R, \epsilon_{b}$ and $\epsilon_{q}$, where $\epsilon_{b}$ and $\epsilon_{q}$ are the $b$-tagging efficiency for heavy flavor and light flavor obtained from QCD data, respectively. $R$ is measured to be $0.98 \pm 0.04$. We also obtained $R>0.85$ at $95 \%$ confidence level. Figure 9 shows the limit bands obtained from the Feldman-Cousins method. The limit bands constrain the true value of $\mathrm{R}$ given the observation in data $R_{o b s}$ and result from the distribution of the profile likelihood obtained from the pseudo-experiments used to compute the lower endpoint of the interval on R.

\section{Top quark charge}

The top quark is the electroweak isospin partner of the bottom quark and has charge of $+2 / 3 \mathrm{e}$ in the SM. The analysis is performed in muon+jet decay mode with $4.6 \mathrm{fb}^{-1}$ data [23]. The top quark charge is assigned using the muons from $W$ boson and soft muons from $B$-hadron decays in $b$-jets. If the charge reconstructed $b$-jet is associated to the leptonic decay branch, the top quark charge is calculated according to $q_{t}=q_{\mu}+q_{b}$. If the charge of the $b$-jet is associated to the hadronic-decay branch, the top quark charge is assigned according to $q_{t}=q_{\mu}-q_{b}$. The number of events selected in categories ' $+2 / 3 \mathrm{e}$ ' and ' $-4 / 3 \mathrm{e}$ ' are shown and compared with the SM simulations in Fig. 10. The wrong charge assignment is measured in a $b$-enriched data sample. The statistical significance of the measurement is given by the p-value for the test statistic A:

$$
\frac{1}{D_{S}} \frac{N_{S M}-N_{X M}-<N_{B G}>D_{B}}{N_{S M}+N_{X M}-<N_{B G}>},
$$

where $D_{S}$ and $D_{B}$ are the dilution of signal and background, respectively, $\left\langle N_{B G}\right\rangle$ is the expected number of backgrounds. The variables $N_{S M}$ and $N_{X M}$ are the number of the SM and exotic model like events, respectively. The exotic top quark $(\mathrm{A}=-1)$ is excluded by this measurement. The measured asymmetry $A_{\text {meas }}$ is $0.97 \pm 0.12$ (stat.) \pm 0.31 (syst.) and agrees very well with the SM expectation of $A=+1$. 


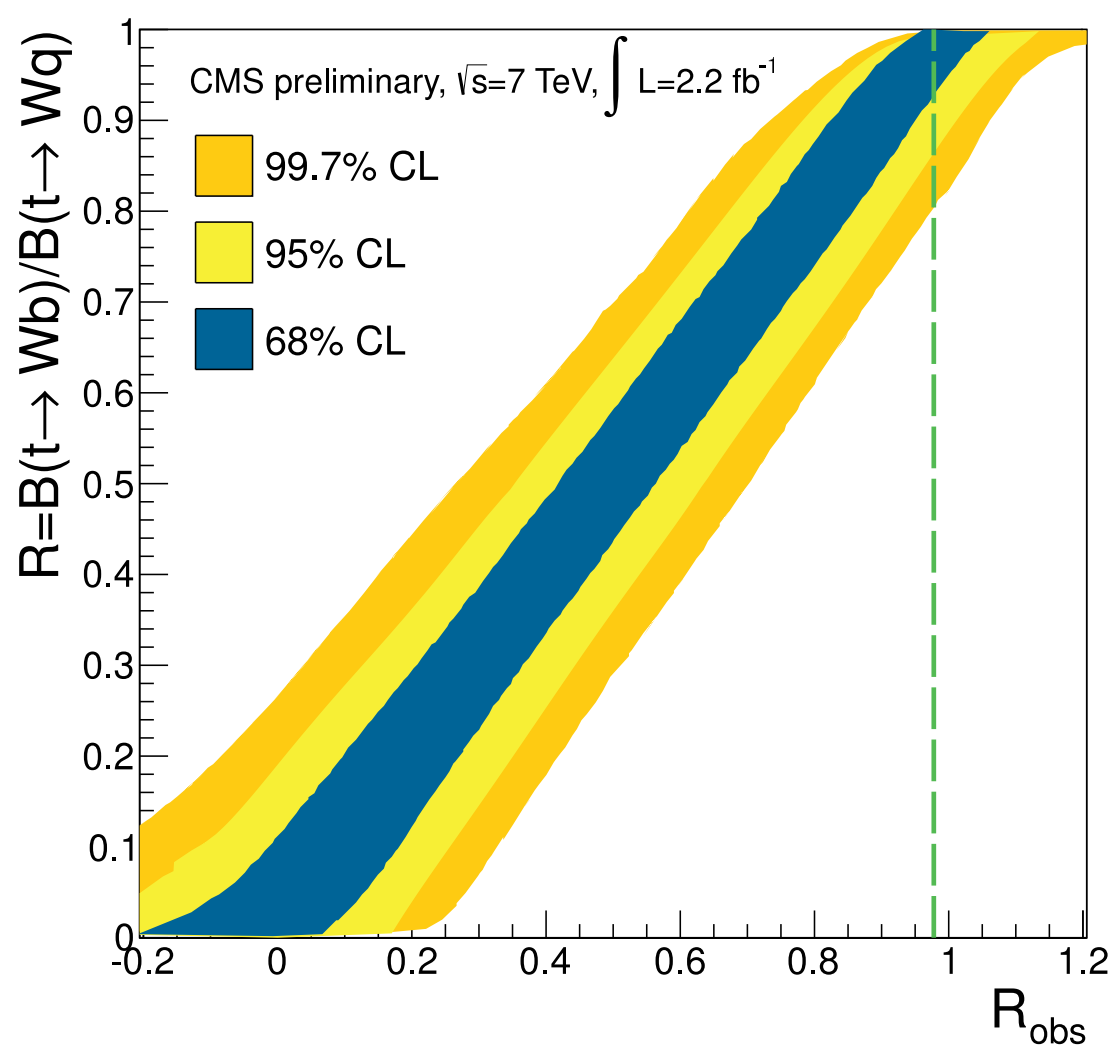

Figure 9. Limit bands at different CL on $R$ with the measured value overlaid as a dashed line. The favored values of $R_{o b s}$ obtained for different CL (horizontal axis) are shown for each true value of $R$ (vertical axis).

\section{$11 W$ polarization}

In the $\mathrm{SM}$, the right-handed helicity fraction of the $W\left(\mathrm{f}_{R}\right)$ is zero at leading order due to helicity suppression assuming $b$ quark mass $=0$. The left-handed helicity fraction $\left(\mathrm{f}_{L}\right)$ and longitudinal fraction $\left(\mathrm{f}_{0}\right)$ are predicted to be approximately $30 \%$ and $70 \%$, respectively. The $W$ boson helicity can be measured using the angular distributions of top decay products. The helicity angle $\left(\theta^{*}\right)$ between lepton three-momentum in the $W$ rest frame and the $W$ momentum in the top rest frame is distributed differently depending on the $W$ helicity. This analysis is performed with the data $2.2 \mathrm{fb}^{-1}$ in lepton+jet decay mode [24]. The $t \bar{t}$ event is reconstructed via a constrained kinematic fit using Lagrange Multipliers. Only events with at least four jets and exactly one lepton are used to reconstruct the masses of the $W$ bosons and top quarks. The $W$ helicity fraction is extracted by likelihood fitting to the angle $\left(\theta^{*}\right)$ distribution. The $\mathrm{f}_{R}$ is measured to be $0.040 \pm 0.035$ (stat.) \pm 0.044 (syst.). The measured fraction can also be used to constrain anomalous $W t b$ couplings. Figure 11 shows the regions of the real component anomalous couplings $g_{L}, g_{R}$ plane allowed at $68 \%$ and $95 \% \mathrm{CL}$. 


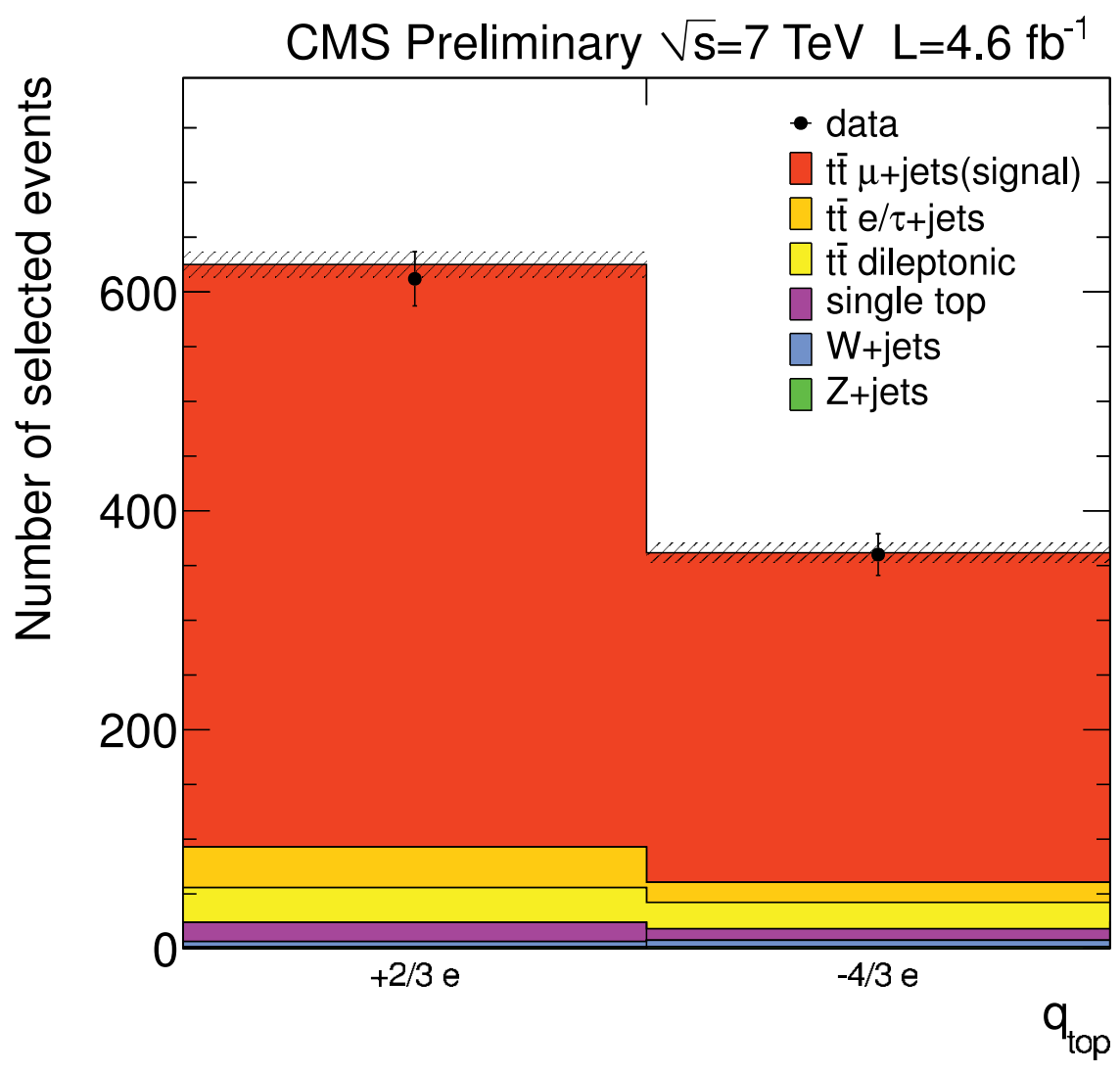

Figure 10. Charge assigned to the top-quark in the selected $t \bar{t}$ events. The data are compared to the standard model predictions.

\section{$12 t \bar{t}$ resonance}

New physics can manifest itself as a $t \bar{t}$ resonance, generically called $Z^{\prime}$, decaying into top pairs are predicted by several models. A narrow topcolor leptophobic $t \bar{t}$ resonance is excluded for masses below about $900 \mathrm{GeV}$ by Tevatron. Searching for new physics in $M_{t \bar{t}}$ are performed in dilepton and lepton+jet decay mode. In dilepton decay mode, neural network (NN) method is used to improve the significance [25] while in lepton+jet decay mode, full event reconstruction method is used fitting together in different number of jets and $b$-tagged jets bins [26]. No significant excess of events above $\mathrm{SM}$ expectations is seen. A leptophobic topcolor $Z^{\prime}$ with width (1.2\%) much less than the detector resolution is excluded for masses below $1.3 \mathrm{TeV}$ in lepton+jets decay mode and $1.1 \mathrm{TeV}$ in dilepton decay mode. In lepton+jets decay mode, a $Z^{\prime}$ with width $10 \%$ of its mass is excluded below $1.7 \mathrm{TeV}$ and a KK gluon is also excluded with a mass below $1.4 \mathrm{TeV}$. 


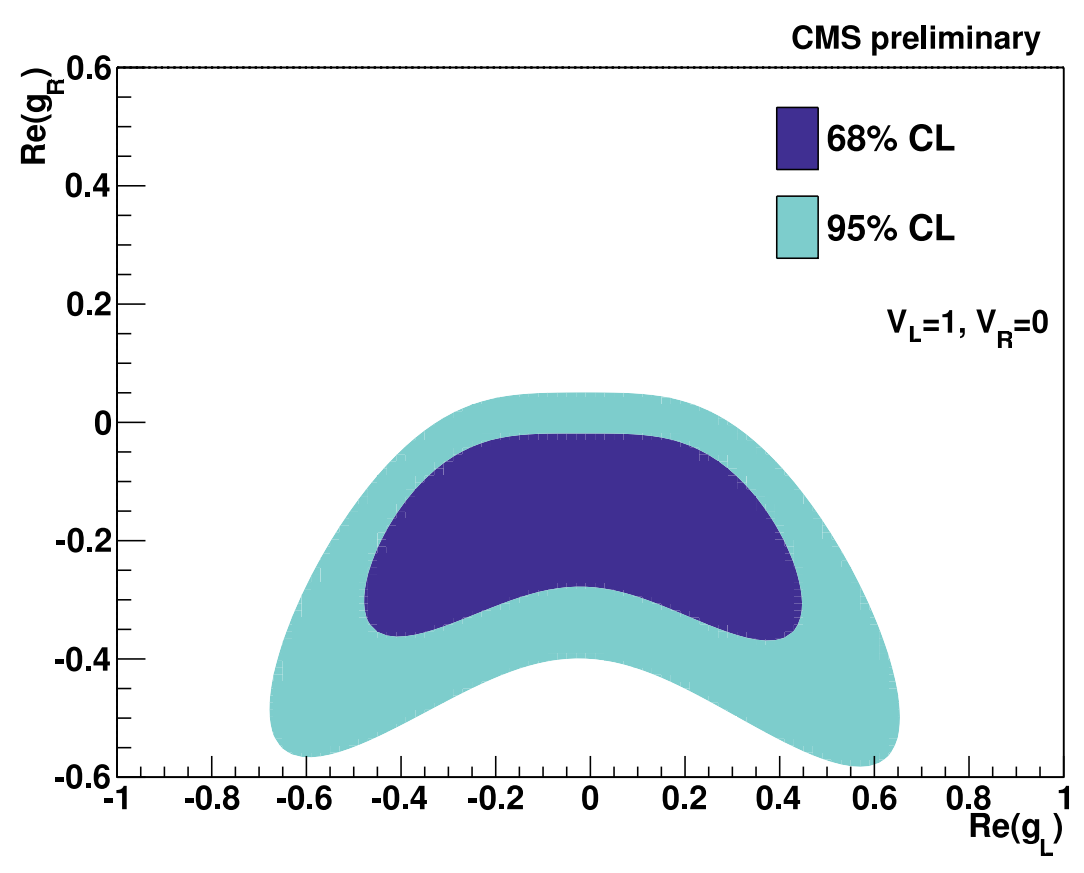

Figure 11. Limits on the real components of the anomalous couplings $g_{L}, g_{R}$ at 68 and $95 \% \mathrm{CL}$, for $V_{L}=1$ and $V_{R}=0$.

\section{Charge asymmetry measurement}

Tevatron has observed deviations in forward-backward measurements. In particular, CDF has observed $3.4 \sigma$ deviation with respect to SM above $450 \mathrm{GeV}$. The deviation could be explained by possible exchange of new particles in t-channel. The charge asymmetry variable is sensitive to this additional production mode. Unlike Tevatron, it is hard to measure in pp collision due to the symmetric initial state of pp collisions at the LHC. Therefore, the rapidity distributions of top and anti-top quarks are symmetrically distributed around zero. However, it is feasible when we consider the top quark (valence quark) rapidity width is broader than anti-top quark (see quark) width. At CMS, the analysis was previously performed with around $1 \mathrm{fb}^{-1}$ showing no deviations [27]. The difference of the absolute rapidity between top quark and anti-top quark $\Delta(|y|)=\left|y_{t}\right|-\left|y_{\bar{t}}\right|$ is used for a new analysis with an integrated luminosity of $5.0 \mathrm{fb}^{-1}$ [28]. Event selection follows the lepton+jets typical analysis requiring 4 jets and one $b$-tagging.

In the SM, it is possible to increase or decrease the asymmetry by changing the kinematic boundary conditions. Therefore, it is crucial to measure the asymmetry differentially in kinematic variables that are sensitive to the asymmetry. Three different variables are used in CMS, namely the rapidity, the transverse momentum and the invariant mass of $t \bar{t}$ system, the vectorially summed four-vector of top quark and anti-quark in the laboratory frame. The reasons why these three variables are chosen are the following. The charge asymmetry occurs only in the $q \bar{q}$ initial state and not in the charge symmetric gluon-gluon initial-state. $t \bar{t}$ production through $q \bar{q}$ annihilation is more prominent for events with 
Table 1. The measured inclusive asymmetry at the different stages of the analysis and the corresponding theory prediction from the SM.

\begin{tabular}{ll}
\hline Uncorrected & $0.003 \pm 0.004$ \\
BG-subtracted & $0.001 \pm 0.005$ \\
Final corrected & $0.004 \pm 0.010 \pm 0.012$ \\
\hline Theory prediction $(\mathrm{SM})$ & $0.0115 \pm 0.0006$ \\
\hline
\end{tabular}

the $t \bar{t}$ pair at larger rapidities. The presence of additional hard radiation (ISR or FSR) implies on average higher transverse momenta of the $t \bar{t}$ system, $p_{T, t \bar{t}}$, compared to events without additional hard radiation. Consequently, in events with large values of $p_{T, t \bar{t}}$, the negative contribution from the ISRFSR interference is enhanced, while for events with small values of $p_{T, t \bar{t}}$, the positive contribution from Born and box diagram interference dominates. If heavy particles with different couplings to top quarks and anti-quarks exist, one can expect an effect on the charge asymmetry with rising invariant mass of the $t \bar{t}$ system, $M_{t \bar{t}}$. Thus, $M_{t \bar{t}}$ can be sensitive to new $t \bar{t}$ production modes via heavy exchange particles. But even in pure SM $t \bar{t}$ production, the charge asymmetry is expected to depend on $M_{t \bar{t}}$ since the contribution of $q \bar{q}$ initial state process is enhanced for large values of $M_{t \bar{t}}$.

In order to compare with theory predictions, the result at reconstruction level needs to be corrected for bin migration and efficiency. For bin migration correction, the regularized unfolding method is applied. For the response matrix, POWHEG is used. For the differential measurement, in order to take into account the bin migration not only between the sensitive variable, $\left|y_{t t}\right|$ but also between differential sensitive variables, 2D regularized unfolding method is applied.

Table 1 shows the values of the measured inclusive asymmetry at the different stages of the analysis. Figure 12 shows the distributions of the inclusive and three differential measurements comparing with predictions from the SM calculations and from an effective field theory. The measured charge asymmetry values at CMS are within the uncertainties in agreement with the SM theory predictions.

\section{Conclusion}

We presented measurements of the cross section in top quark pair production and in single top quark production, top quark mass, top quark properties and new physics searches in top quark decays performed at the CMS experiment with various integrated luminosities by the time of ICFP 2012 conference. All measured results are compatible with SM prediction so far. Measurements of cross section are already systematically limited and starting to constrain theory. Improving the systematic uncertainty for top quark mass measurement is expected with better understanding of jet energy scale and $b$-tagging. For charge asymmetry measurement, more statistics are required to confirm or exclude the observation by Tevatron. 
EPJ Web of Conferences
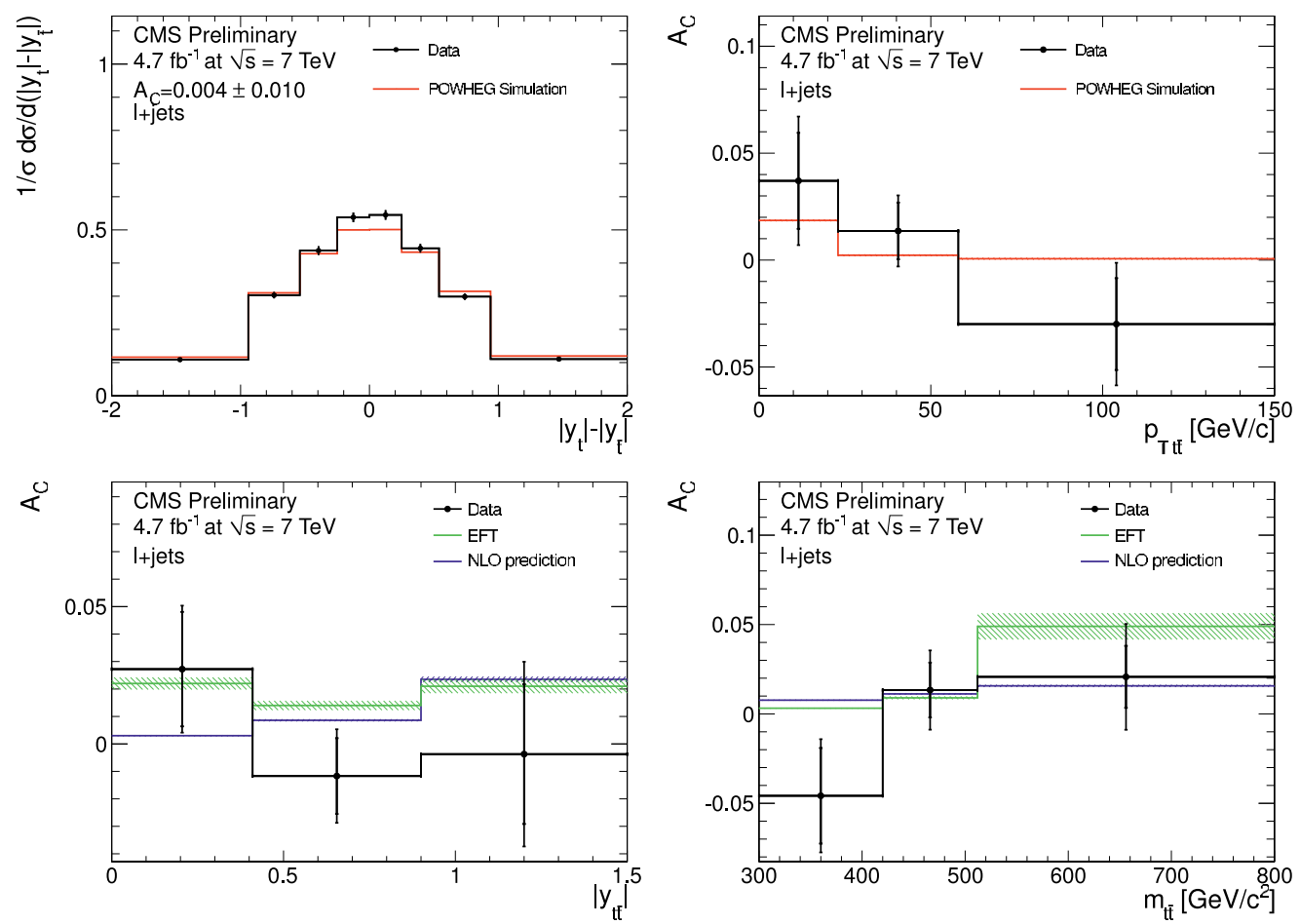

Figure 12. Unfolded $\Delta(|\eta|)$ spectrum (upper-left) and unfolded $\Delta\left(\left|y^{2}\right|\right)$ spectrum (upper-right). The NLO SM prediction is also shown. Raw charge asymmetries for $\Delta(|\eta|)$ (lower-left) and $\Delta\left(\left|y^{2}\right|\right)$ (lower-right) as a function of reconstructed $t \bar{t}$ system mass at CMS. 


\section{References}

[1] CMS Collaboration, "The CMS experiment at the CERN LHC", JINST 3:S08004,2008.

[2] CMS Collaboration, "Performance of $\tau$-lepton reconstruction and identification in CMS", JINST 7 (2012) 1001, doi:10.1088/1748-0221/7/01/P01001, arXiv:1109.6034.

[3] CMS Collaboration, CMS-PAS-TOP-11-005, http://cdsweb.cern.ch/record/1377323

[4] CMS Collaboration, "First Measurement of the Cross Section for Top-Quark Pair Production in Proton-Proton Collisions at $\sqrt{s}=7$ TeV", Phys. Lett. B 695 (2011) 424.

[5] CMS Collaboration, "Measurement of the $t \bar{t}$ production cross section and the top quark mass in the dilepton channel in pp collisions at $\sqrt{s}=7$ TeV", JHEP 07 (2011) 049.

[6] CMS Collaboration, CMS-PAS-TOP-11-004, https://cdsweb.cern.ch/record/1446652

[7] CMS Collaboration, CMS-PAS-TOP-11-006, http://cdsweb.cern.ch/record/1371010

[8] CMS Collaboration, CMS-PAS-TOP-11-003, http://cdsweb.cern.ch/record/1386709

[9] CMS Collaboration, "Measurement of the Top-antitop Production Cross Section in pp Collisions at $\sqrt{s}=7 \mathrm{TeV}$ using the Kinematic Properties of Events with Leptons and Jets", Eur. Phys. J. C 71 (2011) 1721.

[10] CMS Collaboration, "Measurement of the $t \bar{t}$ Production Cross Section in pp Collisions at $7 \mathrm{TeV}$ in Lepton + Jets Events Using b-quark Jet Identification", Phys. Rev. D 84 (2011) 092004.

[11] CMS Collaboration, CMS-PAS-TOP-11-007, http://cdsweb.cern.ch/record/1371755

[12] CMS Collaboration, CMS-PAS-TOP-11-024, http://cdsweb.cern.ch/record/1401250

[13] CMS Collaboration, CMS-PAS-TOP-11-013, http://cdsweb.cern.ch/record/1422425

[14] CMS Collaboration, "Measurement of the single top quark $t$-channel cross section in pp collisions at $\sqrt{s}=7 \mathrm{TeV}$ ", Submitted to the Journal of High Energy Physics, http://cdsweb.cern.ch/record/1430743, http://arxiv.org/abs/1209.4533.

[15] CMS Collaboration, "Evidence for associated production of a single top quark and $W$ boson in pp collisions at $7 \mathrm{TeV}$ ", Submitted to Physical Review Letters, http://cdsweb.cern.ch/record/1385552, http://arxiv.org/abs/1209.3489.

[16] CMS Collaboration, "Measurement of the top quark mass in $t \bar{t}$ events with lepton+jets final states in pp collisions at $\sqrt{s}=7 \mathrm{TeV}$ ", Submitted to the Journal of High Energy Physics, http://cdsweb.cern.ch/record/1427762, http://arxiv.org/abs/1209.2319.

[17] CMS Collaboration, "Measurement of the top quark mass in $t \bar{t}$ events with dilepton final states in pp collisions at $\sqrt{s}=7 \mathrm{TeV}$ ", Submitted to the European Physical Journal C, http://cdsweb.cern.ch/record/1427240, http://arxiv.org/abs/1209.2393.

[18] CMS Collaboration, CMS-PAS-TOP-11-018, http://cdsweb.cern.ch/record/1478194

[19] CMS Collaboration, CMS-PAS-TOP-11-008, http://cdsweb.cern.ch/record/1387001

[20] CMS Collaboration, "Measurement of the mass difference between top and anti-top quarks", Submitted to the Journal of High Energy Physics, http://cdsweb.cern.ch/record/1376668, 
http://arxiv.org/abs/arXiv:1204.2807.

[21] CMS Collaboration, "Search for flavor changing neutral currents in top quark decays in pp collisions at $7 \mathrm{TeV}$ ", http://cdsweb.cern.ch/record/1429971, http://arxiv.org/abs/arXiv:1208.0957

[22] CMS Collaboration, CMS-PAS-TOP-11-029, http://cdsweb.cern.ch/record/1429972

[23] CMS Collaboration, CMS-PAS-TOP-11-031, http://cdsweb.cern.ch/record/1429970

[24] CMS Collaboration, CMS-PAS-TOP-11-020, http://cdsweb.cern.ch/record/1423370

[25] CMS Collaboration, CMS-PAS-TOP-11-010, http://cdsweb.cern.ch/record/1438739

[26] CMS Collaboration, CMS-PAS-TOP-11-009, http://cdsweb.cern.ch/record/1429634

[27] CMS Collaboration, "Measurement of the charge asymmetry in top-quark pair production in proton-proton collisions at $\sqrt{s}=7 \mathrm{TeV}$ ", Phys. Lett. B 709 (2012) 28.

[28] CMS Collaboration, "Inclusive and differential measurements of the $t \bar{t}$ charge asymmetry in proton-proton collisions at $7 \mathrm{TeV}$ ", Submitted to Phys. Lett. B, http://cdsweb.cern.ch/record/1428152, http://arxiv.org/abs/arXiv:1207.0065. 